\title{
Circular RNA circSLC8A1 acts as a sponge of miR-130b/miR-494 in suppressing bladder cancer progression via regulating PTEN
}

Qun Lu ${ }^{1 \dagger}$, Tianyao Liu ${ }^{1 \dagger}$, Huijin Feng ${ }^{2 \dagger}$, Rong Yang ${ }^{1}$, Xiaozhi Zhao ${ }^{1}$, Wei Chen ${ }^{1}$, Bo Jiang ${ }^{1}$, Haixiang Qin ${ }^{1}$, Xu Guo ${ }^{2}$, Minghui Liu', Limin $\mathrm{Li}^{2}$ and Hongqian Guo ${ }^{1 *}$ (i)

\begin{abstract}
Background: Circular RNAs (circRNAs) are a novel class of endogenous noncoding RNAs formed by a covalently closed loop, and increasing evidence has revealed that circRNAs play crucial functions in regulating gene expression. CircSLC8A1 is a circRNA generated from the SLC8A1 gene. Currently, the role and underlying molecular mechanisms of circSLC8A1 in bladder cancer remain unknown.

Methods: The differentially expressed circRNAs were identified from RNA-sequencing data, and circSLC8A1 was determined as a new candidate circRNA. GRT-PCR was used to detect the expression of circRNAs, miRNAs and mRNAs in human tissues and cells. RNA pull-down assay and luciferase reporter assay were used to investigate the interactions between the specific circRNA, miRNA and mRNA. The effects of circSLC8A1 on bladder cancer cells were explored by transfecting with plasmids in vitro and in vivo. The expression of PTEN was detected by Western blot. The biological roles were measured by wound healing assay, transwell assay, and CCK-8 assay.
\end{abstract}

Results: In the present study, we found that circSLC8A1 was down-regulated in bladder cancer tissues and cell lines, and circSLC8A1 expression was associated with the pathological stage and histological grade of bladder cancer. Over-expression of circSLC8A1 inhibited cell migration, invasion and proliferation both in vitro and in vivo. Mechanistically, circSLC8A1 could directly interact with miR-130b/miR-494, and subsequently act as a miRNA sponge to regulate the expression of the miR-130b/miR-494 target gene PTEN and downstream signaling pathway, which suppressed the progression of bladder cancer.

Conclusions: CircSLC8A1 acts as a tumor suppressor by a novel circSLC8A1/miR-130b, miR-494/PTEN axis, which may provide a potential biomarker and therapeutic target for the management of bladder cancer.

\section{Background}

Bladder cancer is the most common malignancy of the urinary system and is one of the most prevalent malignancies worldwide [1]. In China, the mortality and morbidity of bladder cancer ranked first among all the tumors of urinary system [2]. Bladder cancer can be classified into two types according to the depth of tumor invasion: non-muscle

\footnotetext{
* Correspondence: dr.ghq@nju.edu.cn

${ }^{\dagger}$ Qun Lu, Tianyao Liu and Huijin Feng contributed equally to this work. 'Department of Urology, Drum Tower Hospital, Medical School of Nanjing University, Institute of Urology, Nanjing University, 321 Zhongshan Road, Nanjing 210008, Jiangsu, China

Full list of author information is available at the end of the article
}

invasive tumor $(70 \sim 80 \%)$ and muscle-invasive tumor (20 30\%) [3].For the patients with muscle-invasive bladder cancer, the occurrence of metastasis is more frequent, and the prognosis is poorer [4]. Even in those muscle-invasive bladder cancer patients who receive optimal treatment with surgery and chemotherapy, the 5-year overall survival rate is only $60 \%$ due to distant metastasis [5]. Therefore, it is of great clinical significance to clarify the molecular mechanisms that drive the progression of bladder cancer, which will help to develop more effective anticancer therapies.

Circular RNA (circRNA) is a novel class of endogenous noncoding RNA molecules generally characterized

(c) The Author(s). 2019 Open Access This article is distributed under the terms of the Creative Commons Attribution 4.0 International License (http://creativecommons.org/licenses/by/4.0/), which permits unrestricted use, distribution, and 
by a covalently closed loop structure without a $5^{\prime}$ cap and a 3'poly A tail [6]. Unlike linear RNAs, circRNAs usually originate from back splicing events of exons or introns. Reversed complementary sequences including inverted repeated Alu pairs and exon skipping are essential to circRNA formation [7, 8]. Salient features of circRNAs include significant stability, high abundance, evolutionary conservation, and tissue-specific expression $[9,10]$. Although circRNAs were reported many years ago [11], these molecules were first considered as products of splicing errors [12]. Genome-wide analyses of RNA sequencing data have identified large amounts of circRNAs and proven that they are endogenous, abundant and conserved in mammalian cells, suggesting specific roles of circRNAs in cellular physiology [6, 9]. Many circRNAs have been demonstrated to have critical roles in the carcinogenesis and development of cancers $[13,14]$.

MicroRNAs (miRNAs) are endogenous small noncoding RNA molecules (19-22 nucleotides in length) that negatively modulate the expression of protein-coding genes through binding to the specific sequence of genes [15]. Increasing evidence suggests that miRNAs are aberrantly expressed in bladder cancer and they play significant roles in the tumorigenesis, development, and metastasis of cancer [16]. Multiple properties of circRNAs have been identified in recent years, among which the role of "miRNA sponges" was most frequently discussed since some circRNAs possess miRNA binding sites [17]. CircRNAs sequester miRNAs to terminate the regulation of their target genes [18]. Currently, there are few reports describing the role of circRNAs in bladder cancer [19-21]. The biological functions of circRNAs in bladder cancer remain largely unknown and require further investigation.

Here, we identified a circRNA derived from the SLC8A1 gene, termed circSLC8A1. The expression of circSLC8A1 was significantly down-regulated in bladder cancer tissues and cell lines, and it was positively correlated with the clinical stage and grade of bladder cancer. Therefore, we presented a hypothesis that circSLC8A1 might be involved in the progression of bladder cancer, via sponging miR-130b and miR-494 to influence the expression of PTEN. Collectively, circSLC8A1 may serve as a promising target for bladder cancer treatment.

\section{Methods}

\section{Cell lines and human tissues}

The human bladder cancer cell lines (5637, T24, J82, EJ, UMUC, and RT4) and the human urothelial epithelial cell line (SV-HUC-1) were purchased from the Shanghai Institute of Cell Biology, Chinese Academy of Sciences (Shanghai, China). The cells were cultured in RPMI 1640 medium supplemented with $10 \%$ fetal bovine serum (FBS, Gibco, Carlsbad, CA, USA), 100 units $/ \mathrm{ml}$ penicillin and $100 \mu \mathrm{g} / \mathrm{ml}$ streptomycin in a humidified atmosphere containing 5\% CO2. The bladder cancer specimens and paired normal adjacent tissues were obtained from patients undergoing a surgical procedure at the Affiliated Drum Tower Hospital of Nanjing University Medical School (Nanjing, China). All the patients provided written consent, and the Ethics Committee from Nanjing University approved all aspects of this study. Tissue fragments were immediately frozen in liquid nitrogen at the time of surgery and stored at $80^{\circ} \mathrm{C}$.

\section{RNA isolation and quantitative RT-PCR (qRT-PCR)}

Total RNA was extracted from cultured cells and human tissues using Trizol reagent (Sigma, St. Louis, MO, USA) according to the manufacturer's instructions. Reverse transcription of mRNA and miRNA was conducted using random primers and stem-loop primers in TaKaRa system (Dalian, China), respectively. Real-time PCR was performed using a TaqMan Universal Master Mix II kit on an Applied Biosystems 7500 Sequence Detection System (Applied Biosystem). The levels of target genes were calculated based on the cycle threshold $(\mathrm{Ct})$ values compared to a reference gene using the formula $2^{-\Delta \Delta \mathrm{Ct}}$. GAPDH mRNA and U6 snRNA were used as references for mRNA and miRNA, respectively. The details of primers were listed in Additional file 4: Table S2.

\section{Protein extraction and Western blotting}

The cells and tissues were lysed in ice-cold RIPA lysis buffer (Beyotime, Shanghai, China) supplemented with $1 \%$ PMSF, incubated on ice for $30 \mathrm{~min}$ and then centrifuged for $10 \mathrm{~min}\left(12,000 \times \mathrm{g}, 4^{\circ} \mathrm{C}\right)$. The supernatant was collected, and the protein concentration was calculated using a Pierce BCA protein assay kit (Thermo Scientific, Rockford, IL, USA). The PTEN protein levels were analyzed by Western blotting with an anti-human PTEN antibody (9559, Cell Signaling Technology, MA, USA). The protein levels were normalized by probing the same blots with a $\beta$-actin antibody (05-0079, AbMax, Beijing, China).

\section{Plasmid construction and siRNA interference assay}

To construct circSLC8A1 over-expression plasmids, human circSLC8A1 cDNA was synthesized and cloned into a pLVX-cir vector by Genomeditech (Shanghai, China), which contained a front circular frame and a back circular frame. An empty plasmid served as the negative control. Two siRNA sequences were synthesized by GenePharma (Shanghai, China). A scrambled siRNA was synthesized as a negative control. Transfection was carried out using Lipofectamine 3000 (Invitrogen) 
according to the manufacturer's instructions. Total RNA and protein were collected $48 \mathrm{~h}$ after transfection.

\section{Wound healing assay}

Cells were seeded in 6-well plates with $5 \times 10^{5}$ cells per well. Then, a wound was made by using a $200 \mu \mathrm{l}$ pipette tip on the cell monolayer and photographs were taken at the appropriate time to estimate the area occupied by migratory cells.

\section{Transwell assay}

Transwell (Costar, New York, NY, USA) assay was used to evaluate the invasion and migration capacities of bladder cancer cells in vitro. Cells at a concentration of $1 \times 10^{5}$ cells in $500 \mu \mathrm{l}$ of serum-free medium were inoculated in the upper chamber, coated with (invasion assay) or without (migration assay) growth factor reduced Matrigel $^{\bullet}$, and medium containing 10\% FBS was added into the lower chamber as a chemoattractant. After incubation for the appropriate time, cells on the upper surface of the membrane were removed by wiping with a Q-tip, and the invaded or migrated cells were fixed with formaldehyde and stained using $0.5 \%$ crystal violet (Sigma). The numbers of invaded and migrated cells were counted in five randomly selected fields under a microscope.

\section{Cell proliferation assay}

The proliferation of bladder cancer cells was determined using the Cell Counting Kit-8 (CCK-8, Dojindo, Japan) according to the manufacturer's instructions. 5637 and T24 cells were plated at a density of $5 \times 10^{3}$ cells per well in 96-well plates. At the indicated time points, the cells were treated with $10 \mu \mathrm{l}$ of CCK-8 solution (Dojindo, Japan) and incubated in the dark for another $2 \mathrm{~h}$. The absorbance was measured at a wavelength of $450 \mathrm{~nm}$.

\section{Pull-down assay with biotinylated circSLC8A1 probe}

The biotinylated probe was specifically designed to bind to the junction area of circSLC8A1, while the oligo probe was taken as a control. Approximately $1 \times 10^{7}$ cells were harvested and lysed. The circSLC8A1 probe (Tsingke, Wuhan, China) was incubated with streptavidin magnetic beads (Life Technologies, USA) at room temperature for $2 \mathrm{~h}$ to generate probe-coated beads. The cell lysates were incubated with probe-coated beads at $4{ }^{\circ} \mathrm{C}$ overnight. The beads were washed and the bound miRNAs in the pull-down materials were extracted using Trizol reagent and analyzed by qRT-PCR assay.

\section{Pull-down assay with biotinylated miRNA}

Bladder cancer cells were transfected with $50 \mathrm{nM}$ of biotinylated miRNA mimics or nonsense control (Tsingke, Wuhan, China) at 50\% confluence using Lipofectamine
3000 (Invitrogen). The cells were harvested and lysed in lysis buffer $24 \mathrm{~h}$ after transfection. The cell lysates were incubated with washed streptavidin magnetic beads (Life Technologies) for $3 \mathrm{~h}$. The beads were washed and Trizol reagent was used to extract RNA interacting with miRNA. The abundance of circSLC8A1 was evaluated by qRT-PCR analysis.

\section{Fluorescence in situ hybridization (FISH)}

Cy3-labeled probes were specific to circSLC8A1 and fam-labeled probes were specific to miR-130b/miR-494. The probes were designed and synthesized by Genepharma (Shanghai, China), and the signals of the probes were detected by a Fluorescent In Situ Hybridization Kit (Genepharma, Shanghai, China) according to the manufacturer's instructions. The images were acquired on Lei TCS SP8 Laser Scanning Confocal Microscope (Leica Microsystems, Mannheim, Germany).

\section{Luciferase reporter assay}

A sequence containing the presumed binding sites of miR-130b and miR-494 was designed from the human PTEN 3'-UTR. The sequence was inserted into the p-MIR-reporter plasmid. The insertion was confirmed to be correct by sequencing. To test the binding specificity, the sequences that interacted with the miR-130b/miR-494 seed sequence were mutated, and the mutant PTEN 3'-UTR was inserted into an equivalent luciferase reporter. For the luciferase reporter assays, HEK293 cells were cultured in 24-well plates, and each well was transfected with $0.4 \mu \mathrm{g}$ of firefly luciferase reporter plasmid; $0.4 \mu \mathrm{g}$ of a $\beta$-galactosidase ( $\beta$-gal) expression plasmid (Ambion); and equal amounts $(20 \mathrm{pmol})$ of mim-miR-130b/mimmiR-494, or mim-miR-control using Lipofectamine 3000 (Invitrogen). The $\beta$-gal plasmid was used as a transfection control. Twenty-four hours after transfection, the cells were assayed using a luciferase assay kit (Promega, Madison, WI, USA).

\section{Immunohistochemistry (IHC)}

The primary antibody used to detect PTEN was purchased from Cell Signaling Technology (Beverly, MA, USA), and the primary antibodies used to detect Akt, p-Akt, and MMP-9 were purchased from Proteintech Group (Chicago, IL, USA). The immunostaining images were captured using a microscope (Leica, Germany). The immunoreactivity in each tissue section was assessed by at least two pathologists. The degree of positivity was determined according to the percentage of positive tumor cells.

\section{Establishment of tumor xenografts in mice}

Four-week-old female nude mice were purchased from the Model Animal Research Center at Nanjing 
University (Nanjing, China) and maintained under specific pathogen-free conditions at Nanjing University. The mice were randomly divided into 2 groups and subcutaneously injected with T24 cells $\left(5 \times 10^{6}\right.$ cells per mouse, 6 mice per group) that were stably transfected with circSLC8A1 plasmids or control vector. Tumor growth was monitored every week by measuring the width (W) and length $(\mathrm{L})$ with calipers, and the volume $(\mathrm{V})$ of the tumor was calculated using the formula $\mathrm{V}=\left(\mathrm{W}^{2} \times \mathrm{L}\right) / 2$. The mice were sacrificed after four weeks. The tumors were excised, and the tumor weight was measured. Portions of the tumor samples were used for total RNA extraction, and the remainder were fixed in $4 \%$ paraformaldehyde for $24 \mathrm{~h}$ and then processed for hematoxylin and eosin $(\mathrm{H} \& \mathrm{E})$ staining as well as IHC staining.

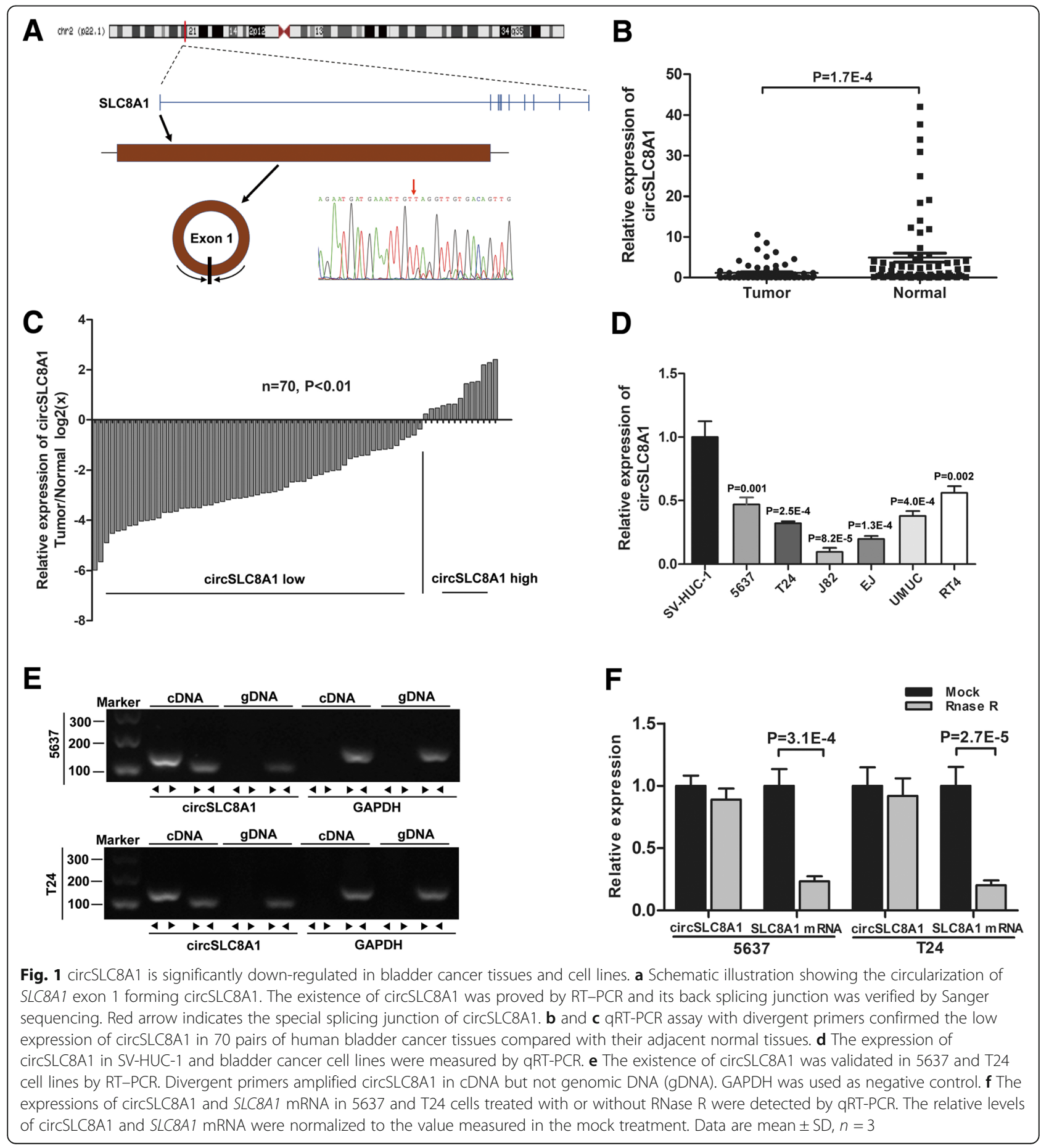




\section{Statistical analysis}

Data are presented as the mean \pm standard deviation (SD) from three independent experiments unless otherwise noted. A paired $t$-test was used to analyze the differences in circSLC8A1 and miR-130b/miR-494 levels between cancer tissues and corresponding normal tissues. Other differences between the two groups were analyzed using the Student's $t$-test or Chi-square test. The Pearson's correlation coefficient analysis was used to analyze the correlations. A $p$-value $<0.05$ was considered statistically significant.

\section{Results}

circSLC8A1 (hsa_circ_0000994) is significantly downregulated in bladder cancer

To identify the role of circRNAs in the development of bladder cancer, differentially expressed circRNAs were studied from the RNA sequencing data of a previous study [19]. Among the differentially expressed circRNAs, downregulated circRNAs are more common than up-regulated circRNAs. We screened the down-regulated circRNAs with an average normal tissue read count of more than 100 and then sorted them by fold change. The five most downregulated circRNAs were validated using qRT-PCR in 20 pairs of bladder cancer tissues and matched adjacent normal tissues, and the details of five circRNAs were listed in Additional file 3: Table S1. The results showed that two of the five circRNAs were significantly down-regulated in bladder cancer tissues, and circSLC8A1 (hsa_circ_0000994) was the most down-regulated circRNA according to the qRT-PCR results (Additional file 1: Figure S1). circSLC8A1 arose from the SLC8A1 gene and consisted of the head-totail splicing of exon 1 (1832 bp) (Fig. 1a). According to the RNA-sequencing result, circSLC8A1 was down-regulated in bladder cancer tissues $(\log 2 \mathrm{FC}=5.28)$. Moreover, we also found that circSLC8A1 was down-regulated in bladder cancer from another RNA-sequencing data set $(\log 2 \mathrm{FC}=$ 3.31) [22], and circSLC8A1 is abundant in early-stage bladder cancer tissues [23]. Subsequently, we confirmed the head-to-tail splicing in the RT-PCR product of circSLC8A1 with the expected size by Sanger sequencing (Fig. 1a). To investigate the expression of circSLC8A1 in bladder cancer, we detected the expression level of circSLC8A1 in 70 pairs of bladder cancer tissue and normal bladder tissue, and the results confirmed that circSLC8A1 had significant low expression in bladder cancer tissues (Fig. 1b). Notably, paired analysis of bladder cancer tumor samples showed that the expression of circSLC8A1 was decreased in more than $80 \%$ of all the patient samples (Fig. 1c). Also, the correlation analysis showed that circSLC8A1 expression was associated with clinicopathological features including the pathological stage and histological grade (Table 1). Additionally, circSLC8A1 showed a lower expression in 6 bladder cancer cell lines (5637, T24, J82, EJ, UMUC, and RT4) compared to SV-HUC-1, which is a normal urothelial cell line
Table 1 Correlations between the expression of circSLC8A1 and clinicopathological features in 70 bladder cancer patients

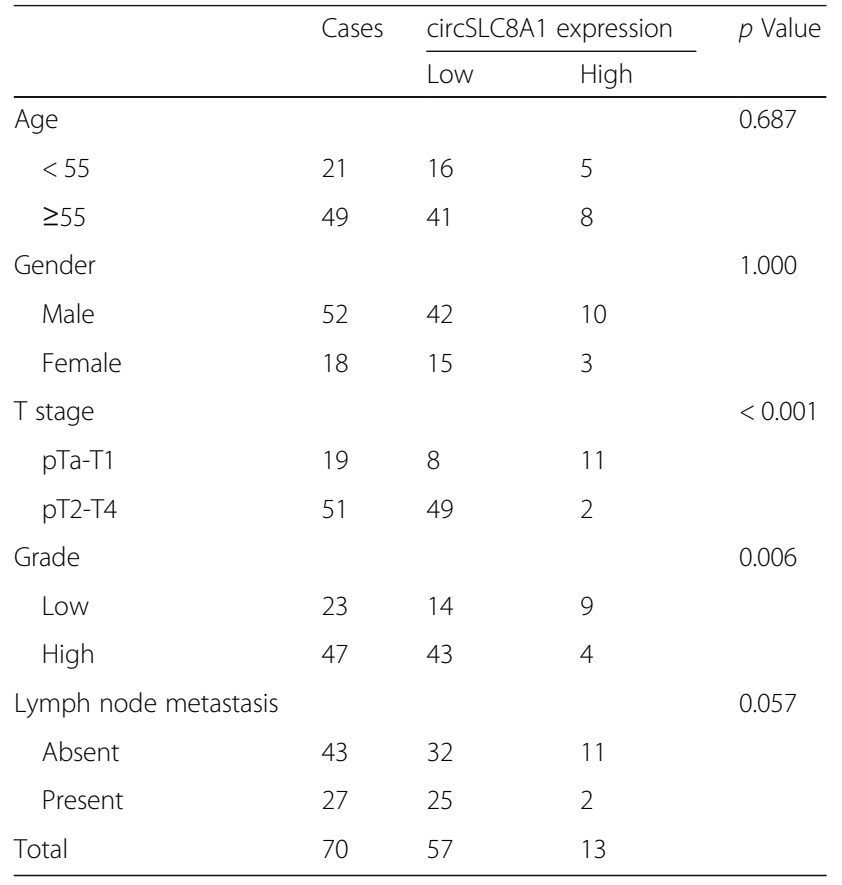

(Fig. 1d). Next, we designed convergent primers to amplify SLC8A1 mRNA and divergent primers to amplify circSLC8A1. Using cDNA and gDNA (genomic DNA) from 5637 and T24 cell lines as templates, circSLC8A1 was only amplified by divergent primers in CDNA, and no amplification product was observed in gDNA (Fig. 1e). By using qRT-PCR, we further confirmed that circSLC8A1 was resistant to RNase R, while SLC8A1 mRNA was significantly reduced after RNase $\mathrm{R}$ treatment (Fig. 1f).

\section{Over-expression of circSLC8A1 inhibits the migration and} invasion of bladder cancer cell

Functional assays were conducted to further validate the role of circSLC8A1 in bladder cancer progression. Given that circSLC8A1 is down-regulated in bladder cancer tissues and cell lines in our study, we transfected the circSLC8A1 expression construct into 5637 and T24 cells, and the expression of circSLC8A1 was significantly increased (Fig. 2a). Meanwhile, the expression of SLC8A1 mRNA had no significant change (Fig. 2a). Wound healing assay revealed that over-expression of circSLC8A1 significantly inhibited cell migration in 5637 and T24 cells (Fig. 2b). Consistently, transwell migration and matrigel invasion assays indicated that the migration and invasion abilities of bladder cancer cell lines were also suppressed by over-expression of circSLC8A1. (Fig. 2c and d). Next, we transfected siRNAs targeting the junction sites of circSLC8A1 into 5637 and T24 cells. These siRNAs significantly decreased the expression of circSLC8A1, but they had no effect on SLC8A1 mRNA (Fig. 2e). The results of 


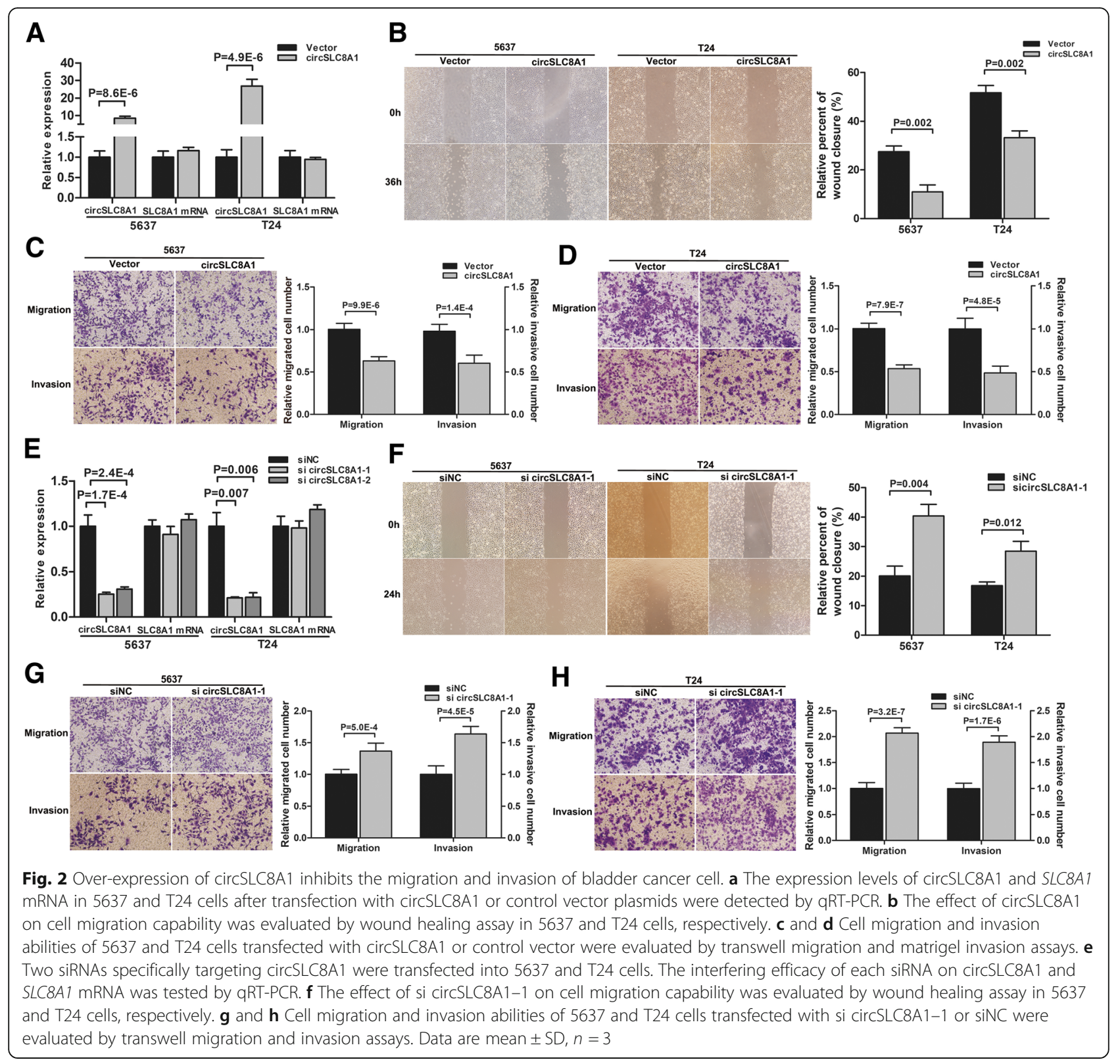

wound healing assay and transwell assay showed that circSLC8A1 knockdown significantly increased the migration and invasion capabilities of 5637 and T24 cells (Fig. 2f-h and Additional file 2: Figure S2). These results suggested that circSLC8A1 played a role as tumor suppressor through inhibiting migration and invasion in bladder cancer cells.

\section{circSLC8A1 acts as a sponge for miR-130b and miR-494 in} bladder cancer cell

It had been reported that circRNAs function as miRNA sponges to regulate miRNA expression [22]. To address whether circSLC8A1 could act as a sponge for miRNAs in bladder cancer cells, we predicted the possible binding miRNAs of circSLC8A1 by using three publicly available prediction tools miRanda (http://www.microrna.org/microrna/home.do), Circinteractome (https://circinteractome.nia.nih.gov/), or RNAhybrid (https:// bibiserv.cebitec.uni-bielefeld.de/rnahybrid/). We selected 7 candidate miRNAs, which were predicted by at least two of the above three prediction tools and previously reported as oncogenic miRNAs. Subsequently, we applied the biotin-labeled probe pull down assay to investigate whether circSLC8A1 could directly bind these candidate miRNAs. The biotin-labeled probe was verified to pull down circSLC8A1 in 5637, T24 and SV-HUC-1 cell lines (Fig. 3a and b). The levels of the 7 candidate miRNAs were detected, and the results revealed that miR-130b and miR-494 were the only two miRNAs that were abundantly 


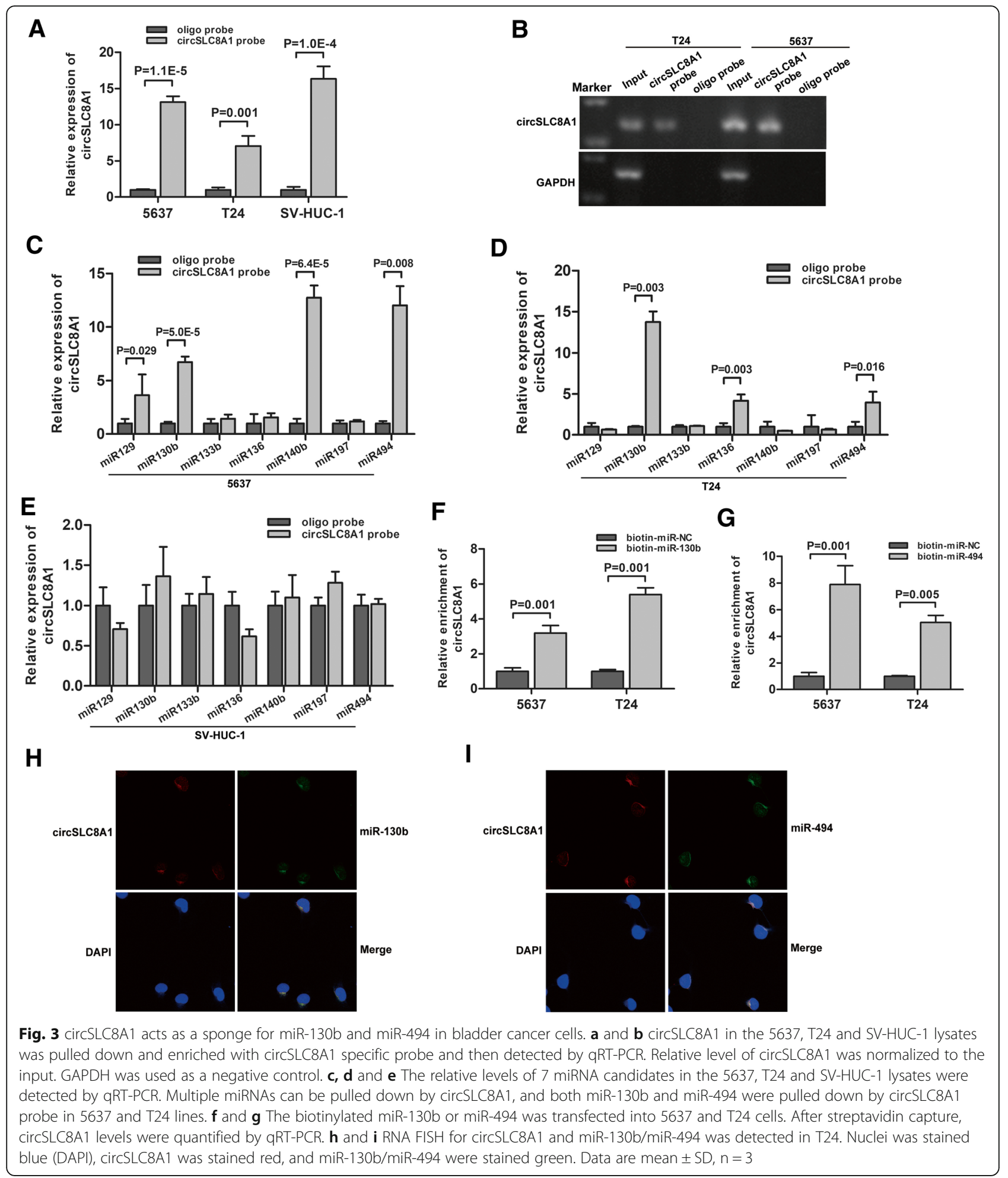

pulled down by circSLC8A1 in both 5637 and T24 cells (Fig. 3c, d and e). To further confirm the sponge effect of circSLC8A1, we applied biotin-labeled miR$130 \mathrm{~b}$ and miR-494 mimics to verify the direct binding of the miRNAs and circSLC8A1. The results showed that biotin-labeled miR-130b/miR-494 captured more circSLC8A1 than the biotin-labeled negative control (Fig. 3f and g). Moreover, RNA FISH assay revealed that circSLC8A1 and miR-130b/miR-494 were colocalized in the cytoplasm (Fig. $3 \mathrm{~h}$ and i). The above 
results indicate that circSLC8A1 can directly bind to miR-130b and miR-494.

\section{miR-130b and miR-494 promote bladder cancer progression through targeting PTEN}

On the basis of the interaction of circSLC8A1 and miR130b/miR-494, we next assessed the potential functional roles of miR-130b and miR-494 in bladder cancer. The result of qRT-PCR showed that miR-130b and miR-494 were up-regulated in bladder cancer tissues compared with normal bladder tissues (Fig. 4a and b, Table 2). Correlation analysis revealed a negative correlation between the expression of circSLC8A1 and miR-130b or miR-494 (Fig. 4c and d). Enforced expression of miR-130b and

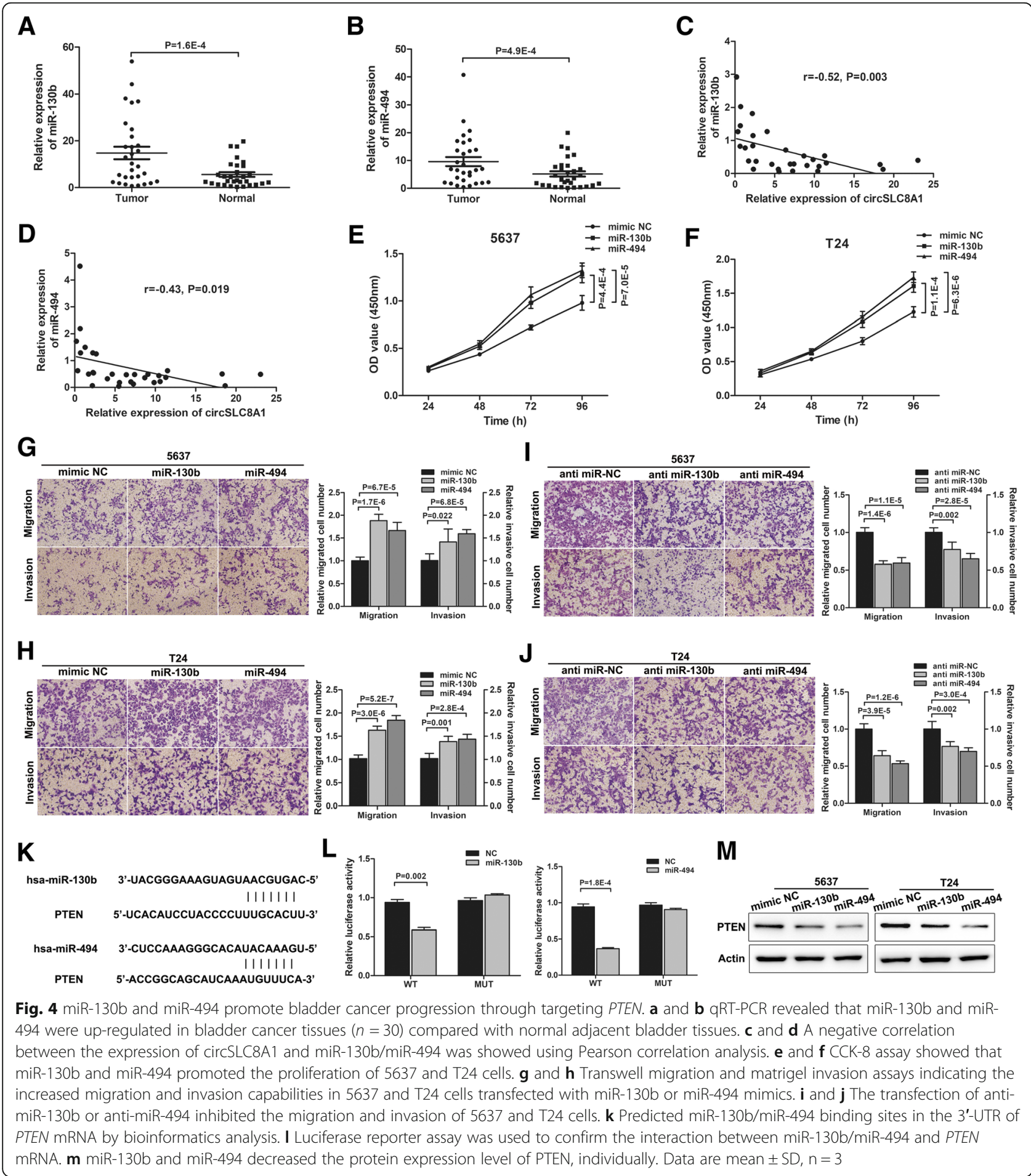


Table 2 Correlations between the expression of miR-130b/miR-494 and clinicopathological features

\begin{tabular}{|c|c|c|c|c|c|c|c|}
\hline & Cases & miR- & & $p$ Value & $\mathrm{miR}-4$ & & $p$ Value \\
\hline & & Low & High & & Low & High & \\
\hline Age & & & & 1.000 & & & 0.952 \\
\hline$<55$ & 11 & 3 & 8 & & 2 & 9 & \\
\hline$\geq 55$ & 19 & 4 & 15 & & 5 & 14 & \\
\hline Gender & & & & 1.000 & & & 0.914 \\
\hline Male & 24 & 6 & 18 & & 5 & 19 & \\
\hline Female & 6 & 1 & 5 & & 2 & 4 & \\
\hline T stage & & & & 0.010 & & & $<0.001$ \\
\hline pTa-T1 & 8 & 5 & 3 & & 6 & 2 & \\
\hline pT2-T4 & 22 & 2 & 20 & & 1 & 21 & \\
\hline Grade & & & & 0.004 & & & 0.047 \\
\hline Low & 10 & 6 & 4 & & 5 & 5 & \\
\hline High & 20 & 1 & 19 & & 2 & 18 & \\
\hline Lymph node metastasis & & & & 0.182 & & & 0.642 \\
\hline Absent & 17 & 6 & 11 & & 5 & 12 & \\
\hline Present & 13 & 1 & 12 & & 2 & 11 & \\
\hline Total & 30 & 7 & 23 & & 7 & 23 & \\
\hline
\end{tabular}

$p<0.05$ represents statistical significance

miR-494 significantly promoted 5737 and T24 proliferation (Fig. 4e and f). Transwell assays showed that overexpression of miR-130b or miR-494 significantly promoted the migration and invasion of 5637 and T24 cells (Fig. $4 \mathrm{~g}$ and $\mathrm{h}$ ). In contrast, transfection of miR-130b/ miR-494 inhibitor obviously suppressed cell migration and invasion of 5637 and T24 cells (Figs. $4 i$ and j). It has been reported that miRNAs post-transcriptionally regulate their target mRNA via sequence-guided recognition. According to TargetScan (http://www.targetscan.org/) and miRanda predictions, we found that miR-130b and miR-494 could both bind to the 3 '-UTR region of PTEN (Fig. 4k). Next, luciferase reporter assays were performed to verify this interaction. The results showed that transfection of miR-130b or miR-494 mimics could strongly reduce the activity of a luciferase reporter carrying the wild-type PTEN 3'-UTR compared to mimic NC. Inversely, the mutated luciferase reporter was unaffected by overexpression of either miR-130b or miR-494 (Fig. 4l). Western blot assays demonstrated that miR-130b or miR-494 mimics could suppress the expression of PTEN (Fig. $4 \mathrm{~m}$ ). These results reveal that $\mathrm{miR}-130 \mathrm{~b} / \mathrm{miR}-494$ can significantly promote the migration, invasion, and proliferation of bladder cancer through targeting PTEN.

circSLC8A1 regulates PTEN expression and inhibits bladder cancer progression via targeting miR-130b and miR-494

To assess whether circSLC8A1 inhibited the progression of bladder cancer cells via miR-130b and miR-494, rescue experiments were conducted by co-transfecting
circSLC8A1 and miR-130b/miR-494 mimics into bladder cancer cells. Transwell matrigel invasion and CCK-8 assays in bladder cancer cells showed that overexpression of circSLC8A1 led to inhibition of the invasion and proliferation capabilities, but this effect could be partly attenuated by ectopic expression of miR-130b or miR494 (Fig. 5a, b and c). Moreover, we found that the expression of PTEN was significantly decreased in the bladder cancer cells co-transfected with circSLC8A1 plasmids and miR-130b/miR-494 mimics, compared with the cells transfected with circSLC8A1 alone (Fig. 5d and e), which agreed with the results of cell function. Additionally, the expression levels of PTEN protein were strikingly lower in bladder cancer specimens compared to adjacent noncancerous specimens (Fig. 5f). The above results demonstrated that circSLC8A1 suppressed bladder cancer progression via sponging miR-130b/miR-494 and regulated PTEN expression.

\section{circSLC8A1 suppresses the growth of bladder cancer tumor in vivo}

To investigate whether over-expression of circSLC8A1 regulates tumor growth in vivo, T24 cells stably transfected with circSLC8A1 or control vector were injected subcutaneously into $\mathrm{BALB} / \mathrm{c}$ nude mice. The tumor volumes were measured weekly after injection. Compared with the control group, the circSLC8A1 over-expression group significantly reduced the growth rate and tumor weight (Fig. 6c and d). In the circSLC8A1 over-expression group, the level of circSLC8A1 was upregulated, whereas 

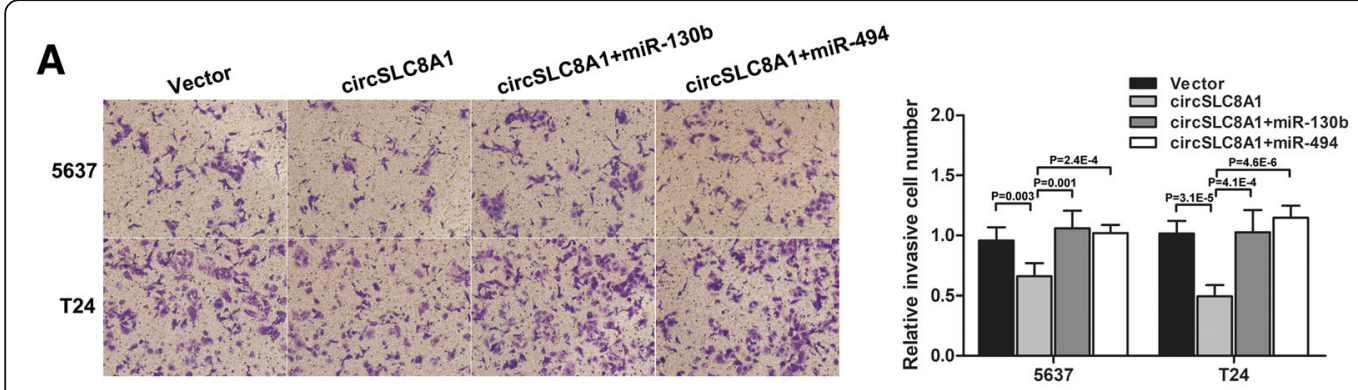

B

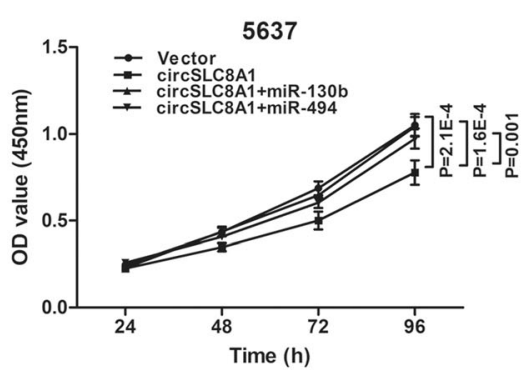

D

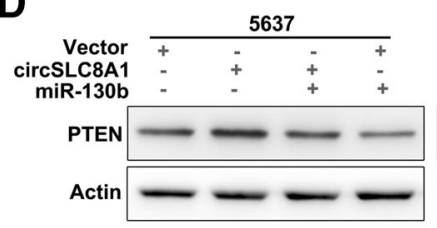

E
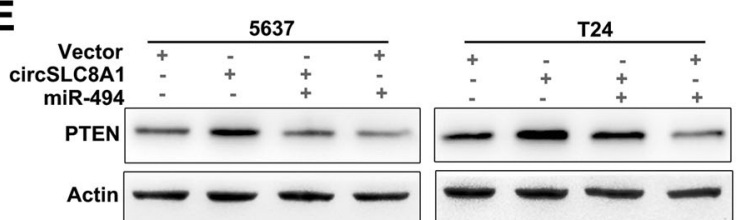

C

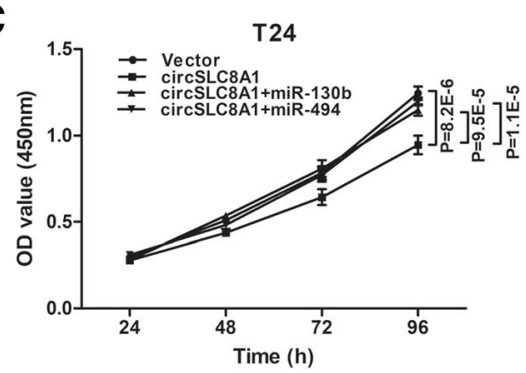

$\mathbf{F}$

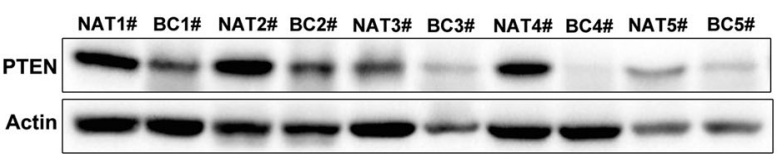

NAT6\# BC6\# NAT7\# BC7\# NAT8\# BC8\# NAT9\# BC9\# NAT10\# BC10\#

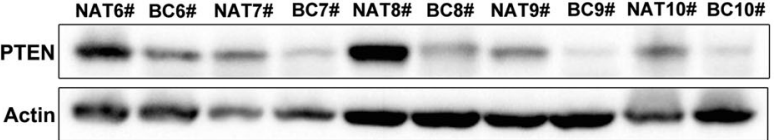

Fig. 5 circSLC8A1 regulates PTEN expression and inhibits bladder cancer progression via targeting miR-130b and miR-494. a, b and c Transwell invasion and CCK-8 assays demonstrating that cirCSLC8A1 inhibited the invasion ability and proliferation of 5637 and T24 cells, and when COtransfected with miR-130b or miR-494, the inhibitory effect was reversed. $\mathbf{d}$ and $\mathbf{e}$ Western blot showed that miR-130b and miR-494 could partly decrease the protein expression level of PTEN which were promoted by circSLC8A1. $\mathbf{f}$ Western blot analysis of PTEN protein in 10 paired human bladder cancer specimens and normal adjacent tissue samples. Data are mean $\pm \mathrm{SD}, \mathrm{n}=3$

the levels of miR-130b and miR-494 were downregulated according to the qRT-PCR results (Fig. 6e). IHC analysis showed that the expression of PTEN was elevated in the tumors by over-expressing circSLC8A1 (Fig. 6f). It is known that PTEN is the negative regulator of the PI3K/ Akt pathway, and MMP-9 protein which is a key factor of cancer invasion is regulated by the PI3K/Akt signaling pathway $[24,25]$. IHC analysis revealed that the expression of phosphorylated Akt (p-Akt) and MMP-9 was inhibited by over-expressing circSLC8A1 (Fig. 6f). These results demonstrate that over-expression of circSLC8A1 efficiently inhibits the growth of bladder cancer in vivo.

\section{Discussion}

In this study, we first confirmed that circSLC8A1 was an important circRNA frequently down-regulated in bladder cancer tissue, and a lower expression of circSLC8A1 was positively correlated with the histological grade and tumor stage. Second, we demonstrated that circSLC8A1 acted as a sponge of miR-130b/miR-494 in suppressing bladder cancer progression via regulating PTEN. Given the importance of the PTEN signaling in bladder cancer progression, our results revealed the function, mechanism and clinical implication of circSLC8A1 in human bladder cancer.

Increasing evidence suggests that circRNAs are not simply the junk-products in pre-mRNA splicing [26]. With the advent of next-generation sequencing, numerous of circRNAs have been identified from various animal genomes, and many of them are highly stable and abundantly expressed. During the past years, a group of circRNAs has been reported to be dysregulated in diverse cancer types, such as hepatocellular carcinoma [22], esophageal squamous cell carcinoma [27], oral 


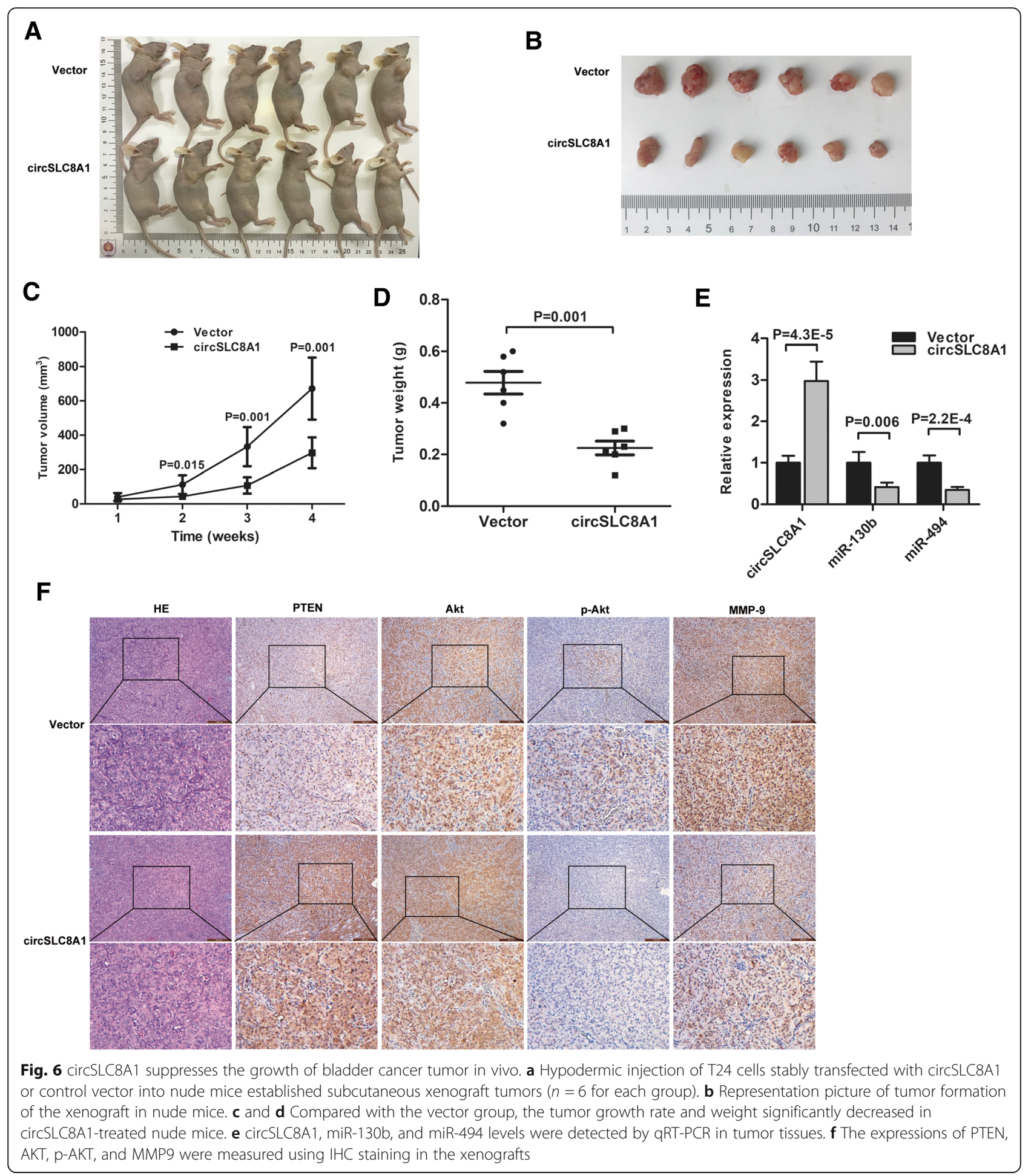

cancer [28], bladder cancer [29], and other types of cancer [30]. Many studies have confirmed that stable transcripts with a host of miRNA-binding sites or miRNA response elements (MREs) could function as miRNA sponges [6], and circRNAs are found to be enriched in functional miRNA binding sites [17].
CircRNAs, which have been regarded as miRNA sponges, have some common characteristics. They are derived from one or more exons of known protein-coding genes through back-splicing [6]. Moreover, the subcellular location of these circRNAs is predominantly in the cytoplasm, which occupies the same space as miRNA. Besides, circRNAs 
harboring more predictive putative miRNA binding sites could be potential candidates for miRNA sponges [22]. It should be noted that not all the circRNAs can act as miRNA sponges. CircRNAs with small length, which are apparently not suitable for miRNA sponges, can be packaged into exosomes and function as cancer biomarkers [31]. Intronic and exon-intron RNAs, which mainly localize in nucleus, have been reported to regulate their parental genes expression via specific RNA-RNA interaction [32].

Due to the sequence conservation, biological stability and tissue specificity of circRNAs, they are thought to be promising biomarkers, therapy targets, and may exert potential functions in the regulation of gene expression $[33,34]$. To better understand the regulatory mechanism of circRNA in this study, we focused on circSLC8A1 which was down-regulated in bladder cancer first. We identified that circSLC8A1, which consists of one exon (1832 bp) from the SLC8A1 gene, mainly located in the cytoplasm. Our miRNA-targeting prediction analysis showed that circSLC8A1 contained multiple miRNA binding sites, suggesting that circSLC8A1 may act as a miRNA sponge. Our results revealed that circSLC8A1 interacted with both miR-130b and miR-494 in bladder cancer cells.

Next, we found that the circSLC8A1-mediated inhibition of invasion and proliferation could be attenuated by enforced overexpression of miR-130b or miR-494. Furthermore, bioinformatics prediction and luciferase reporter assay revealed that miR-130b and miR-494 were critical negative regulators of the PTEN pathway. Lastly, circSLC8A1 could regulate PTEN expression by sponging miR-130b and miR494. Upregulated expression of circSLC8A1 led to the activation of PTEN signaling in bladder cancer, which could be alleviated by miR-130b and miR-494.

PTEN has been widely known as a tumor suppressor gene, and PTEN mutation or deletion is frequently noted in many cancers [35]. The most known function of PTEN is as a negative regulator of the PI3K/Akt pathway, which is a crucial signal transduction pathway for cancer cell growth [36]. Moreover, an integrated study of 131 high grade muscle-invasive bladder cancer samples has revealed dysregulation of the PI3K/Akt signaling pathway in $72 \%$ of cases [37]. The forced expression of PTEN reduced Akt phosphorylation as well as suppressed cell proliferation, migration and invasion $[38,39]$. Additionally, MMP-9 protein, which is a key factor of bladder cancer invasion, is regulated by the PI3K/Akt signaling pathway [25]. IHC analysis of xenografts in our study revealed that the expressions of p-Akt and MMP-9 were inhibited by over-expressing circSLC8A1.

\section{Conclusions}

In conclusion, the results of our study demonstrate that circSLC8A1 is down-regulated in bladder cancer tissues and cell lines, and it is capable of functioning as a sponge for miR-130b and miR-494 to regulate the expression of PTEN. Moreover, we also demonstrate that overexpression of circSLC8A1 can effectively inhibit the progression of bladder cancer via targeting the miR-130b, miR-494/PTEN axis. Our results not only explain the mechanisms of circRNA in regulating bladder cancer cell progression, but also provide a potential biomarker and therapeutic target for the management of bladder cancer.

\section{Additional files}

\begin{abstract}
Additional file 1: Figure S1. Five candidate circRNAs were validated using QRT-PCR in bladder cancer tissues and matched adjacent normal tissues. A to $\mathbf{E}$ The expression levels of the five circRNAs were validated by qRT-PCR in 20 patients. $\mathbf{F}$ The comparison between RNA-sequencing data and qRT-PCR results. The vertical axis showed the mean of the fold change (log2 transformed) of each circRNA measured by qRT-PCR and RNA-sequencing, respectively. (JPG $1781 \mathrm{~kb}$ )
\end{abstract}

Additional file 2: Figure S2. Cell migration and invasion abilities of 5637 and T24 cells transfected with si circSLC8A1-2 or siNC were evaluated. $\mathbf{A}$ The effect of si circSLC8A1-2 on cell migration capability was evaluated by wound healing assay in 5637 and T24 cells, respectively. B and C Cell migration and invasion abilities of 5637 and T24 cells transfected with si circSLC8A1-2 or siNC were evaluated by transwell migration and invasion assays. (JPG $5484 \mathrm{~kb}$ )

Additional file 3: Table S1. Detailed information of five candidate circRNAs. (DOCX $14 \mathrm{~kb}$ )

Additional file 4: Table S2. Primers and RNA sequences used in this study. (DOCX 19 kb)

\section{Abbreviations}

3'-UTR: 3' untranslated region; CCK-8: Cell Counting Kit-8; circRNA: circular RNA; FBS: Fetal bovine serum; H\&E: Hematoxylin and eosin;

IHC: Immunohistochemistry; miRNA: microRNA; qRT-PCR: Quantitative reverse transcription polymerase chain reaction; siRNA: small interfering RNA; $\beta$ gal: $\beta$-galactosidase

\section{Authors' contributions}

HQG and QL conceived and designed the experiments. QL, TYL, HJF, RY and $\mathrm{XZZ}$ participated in the experiments and drafted the manuscript. WC, BJ and $\mathrm{HXQ}$ contributed to the sample collection and interpretation of the data. XG and MHL performed the statistical analysis. HQG and LML revised the manuscript. All authors read and approved the final manuscript.

\section{Funding}

This work was supported by grants from the National Natural Science Foundation of China (81502203, 81772710, 81772727), the National Natural Science Foundation of Jiangsu Province (BK20150097), the Project of Invigorating Health Care through Science, Technology and Education Jiangsu Provincial Key Medical Discipline (ZDXKB2016014), the "Summit of the Six Top Talents" Program of Jiangsu Province (SWYY-084), the Postdoctoral Scientific Research Project of China (2017 M621729), the Postdoctoral Research Foundation of Jiangsu Province (1701021B), and the Nanjing Medical Science and Technique Development Foundation (QRX17139)

\section{Availability of data and materials}

Please contact the corresponding author for all data requests.

\section{Ethics approval and consent to participate}

The research protocol was reviewed and approved by the Ethical Committee of Nanjing Drum Tower Hospital, Medical School of Nanjing University. Written informed consent was obtained from each patient included in the study. 


\section{Consent for publication}

Not applicable.

\section{Competing interests}

The authors declare that they have no competing interests.

\section{Author details}

'Department of Urology, Drum Tower Hospital, Medical School of Nanjing University, Institute of Urology, Nanjing University, 321 Zhongshan Road, Nanjing 210008, Jiangsu, China. ${ }^{2}$ NJU Advanced Institute for Life Sciences, Jiangsu Engineering Research Center for MicroRNA Biology and Biotechnology, Nanjing University, 163 Xianlin Avenue, Nanjing 210023, Jiangsu, China.

Received: 21 December 2018 Accepted: 19 June 2019

Published online: 22 June 2019

\section{References}

1. Antoni S, Ferlay J, Soerjomataram I, et al. Bladder Cancer incidence and mortality: a global overview and recent trends [J]. Eur Urol. 2017;71(1):96-108.

2. Chen W, Zheng R, Baade PD, et al. Cancer statistics in China, 2015 [J]. CA Cancer J Clin. 2016:66(2):115-32.

3. van Rhijn BW, Burger M, Lotan Y, et al. Recurrence and progression of disease in non-muscle-invasive bladder cancer: from epidemiology to treatment strategy [J]. Eur Urol. 2009;56(3):430-42.

4. Dy GW, Gore JL, Forouzanfar MH, et al. Global burden of urologic cancers, 1990-2013 [J]. Eur Urol. 2017;71(3):437-46.

5. Soloway MS. Bladder cancer: Lack of progress in bladder cancer--what are the obstacles? [J]. Nature reviews Urology. 2013;10(1):5-6.

6. Memczak S, Jens M, Elefsinioti A, et al. Circular RNAs are a large class of animal RNAs with regulatory potency [J]. Nature. 2013;495(7441):333-8.

7. Zhang $X O$, Wang $H B$, Zhang $Y$, et al. Complementary sequence-mediated exon circularization [J]. Cell. 2014;159(1):134-47.

8. Vicens Q, Westhof E. Biogenesis of circular RNAs [J]. Cell. 2014;159(1):13-4.

9. Rybak-Wolf A, Stottmeister C, Glazar P, et al. Circular RNAs in the mammalian brain are highly abundant, conserved, and dynamically expressed [J]. Mol Cell. 2015:58(5):870-85.

10. Salzman J, Chen RE, Olsen MN, et al. Cell-type specific features of circular RNA expression [J]. PLoS Genet. 2013;9(9):e1003777.

11. Hsu MT, Coca-Prados M. Electron microscopic evidence for the circular form of RNA in the cytoplasm of eukaryotic cells [J]. Nature. 1979;280(5720):339-40.

12. Cocquerelle C, Mascrez B, Hetuin D, et al. Mis-splicing yields circular RNA molecules [J]. FASEB J. 1993;7(1):155-60.

13. Kristensen LS, Hansen TB, Veno MT, et al. Circular RNAs in cancer: opportunities and challenges in the field [J]. Oncogene. 2018;37(5):555-65.

14. Wang Y, Mo Y, Gong Z, et al. Circular RNAs in human cancer [J]. Mol Cancer. 2017;16(1):25

15. Bartel DP. MicroRNAs: genomics, biogenesis, mechanism, and function [J]. Cell. 2004;116(2):281-97.

16. Yoshino $\mathrm{H}$, Seki $\mathrm{N}$, Itesako $\mathrm{T}$, et al. Aberrant expression of microRNAs in bladder cancer [J]. Nat Rev Urol. 2013;10(7):396-404.

17. Hansen $\mathrm{TB}$, Jensen $\mathrm{TI}$, Clausen $\mathrm{BH}$, et al. Natural RNA circles function as efficient microRNA sponges [J]. Nature. 2013;495(7441):384-8.

18. Sumazin P, Yang $X$, Chiu HS, et al. An extensive microRNA-mediated network of RNA-RNA interactions regulates established oncogenic pathways in glioblastoma [J]. Cell. 2011;147(2):370-81.

19. Li Y, Zheng F, Xiao X, et al. CircHIPK3 sponges miR-558 to suppress heparanase expression in bladder cancer cells [J]. EMBO Rep. 2017;18(9):1646-59.

20. Yang C, Yuan W, Yang $X$, et al. Circular RNA circ-ITCH inhibits bladder cancer progression by sponging miR-17/miR-224 and regulating p21, PTEN expression [J]. Mol Cancer. 2018:17(1):19.

21. Zhong Z, Huang M, LV M, et al. Circular RNA MYLK as a competing endogenous RNA promotes bladder cancer progression through modulating VEGFANEGFR2 signaling pathway [J]. Cancer Lett. 2017:403:305-17.

22. Zheng Q, Bao C, Guo W, et al. Circular RNA profiling reveals an abundant circHIPK3 that regulates cell growth by sponging multiple miRNAs [J]. Nat Commun. 2016:7:11215.

23. Okholm TLH, Nielsen MM, Hamilton MP, et al. Circular RNA expression is abundant and correlated to aggressiveness in early-stage bladder cancer [J]. NPJ genomic medicine. 2017;2:36
24. Tsuruta H, Kishimoto H, Sasaki T, et al. Hyperplasia and carcinomas in Ptendeficient mice and reduced PTEN protein in human bladder cancer patients [J]. Cancer Res. 2006;66(17):8389-96.

25. Cheng JC, Chou CH, Kuo ML, et al. Radiation-enhanced hepatocellular carcinoma cell invasion with MMP-9 expression through PI3K/Akt/NFkappaB signal transduction pathway [J]. Oncogene. 2006;25(53):7009-18.

26. Chen LL. The biogenesis and emerging roles of circular RNAs [J]. Nat Rev Mol Cell Biol. 2016;17(4):205-11.

27. Xia W, Qiu M, Chen R, et al. Circular RNA has_circ_0067934 is upregulated in esophageal squamous cell carcinoma and promoted proliferation [J]. Sci Rep. 2016:6:35576.

28. Chen L, Zhang S, Wu J, et al. circRNA_100290 plays a role in oral cancer by functioning as a sponge of the miR-29 family [J]. Oncogene. 2017;36(32):4551-61.

29. Xie F, Li Y, Wang M, et al. Circular RNA BCRC-3 suppresses bladder cancer proliferation through miR-182-5p/p27 axis [J]. Mol Cancer. 2018;17(1):144.

30. Hansen TB, Kjems J, Damgaard CK. Circular RNA and miR-7 in cancer [J]. Cancer Res. 2013;73(18):5609-12.

31. Li Y, Zheng Q, Bao C, et al. Circular RNA is enriched and stable in exosomes: a promising biomarker for cancer diagnosis [J]. Cell Res. 2015;25(8):981-4.

32. Li Z, Huang C, Bao C, et al. Exon-intron circular RNAs regulate transcription in the nucleus [J]. Nat Struct Mol Biol. 2015;22(3):256-64.

33. Jeck WR, Sorrentino JA, Wang $K$, et al. Circular RNAs are abundant, conserved, and associated with ALU repeats [J]. Rna. 2013;19(2):141-57.

34. Meng $\mathrm{S}$, Zhou $\mathrm{H}$, Feng $\mathrm{Z}$, et al. CircRNA: functions and properties of a novel potential biomarker for cancer [J]. Mol Cancer. 2017;16(1):94.

35. Tamguney T, Stokoe D. New insights into PTEN [J]. J Cell Sci. 2007; 120(Pt 23):4071-9.

36. Morgensztern D, McLeod HL. PI3K/Akt/mTOR pathway as a target for cancer therapy [J]. Anti-Cancer Drugs. 2005;16(8):797-803.

37. Cancer Genome Atlas Research N. Comprehensive molecular characterization of urothelial bladder carcinoma [J]. Nature. 2014; 507(7492):315-22

38. Pan C, Yao G, Liu B, et al. Long noncoding RNA FAL1 promotes cell proliferation, invasion and epithelial-mesenchymal transition through the PTEN/AKT signaling Axis in non-small cell lung Cancer [J]. Cell Physiol Biochem. 2017:43(1):339-52.

39. Li N, Miao Y, Shan Y, et al. MiR-106b and miR-93 regulate cell progression by suppression of PTEN via PI3K/Akt pathway in breast cancer [J]. Cell Death Dis. 2017:8(5):e2796.

\section{Publisher's Note}

Springer Nature remains neutral with regard to jurisdictional claims in published maps and institutional affiliations.

Ready to submit your research? Choose BMC and benefit from:

- fast, convenient online submission

- thorough peer review by experienced researchers in your field

- rapid publication on acceptance

- support for research data, including large and complex data types

- gold Open Access which fosters wider collaboration and increased citations

- maximum visibility for your research: over $100 \mathrm{M}$ website views per year

At BMC, research is always in progress.

Learn more biomedcentral.com/submissions 